\title{
Flint Objects and Bone Object
}

\author{
Lisa Maher
}

According to Hearst Museum records, the following objects were found in tombs from cemeteries $\mathrm{N} 2000$ and N 2500. Mace's original excavation notes, however, do not reference finding knives, chisels, or any flint objects at all in the tombs in these cemeteries. It is possible that these objects actually derive from other cemeteries at Naga ed-Deir, ones where such objects were found, such as $\mathrm{N}$ 100, 500, 1500 , and 3500 .

\section{Flint Catalogue}

Explanation of the Flint Catalogue:

The finds' description of context below is taken from the Catalogue of Tombs. The photograph numbers reflect the original excavator's numbering system.

Material: Flint, bone.

Measurements: Length, width, thickness.

N 2093 (TC) Map C5; photo B 7129, B 7144

[ed.: According to Hearst Museum records, PAHMA 6-14415 is from the large chamber in this burial.]

(1) РАнмА 6-144153 Knife

Unfortunately, this piece could not be located by the Collections Manager in the museum collections for analysis. 
Interior Surface

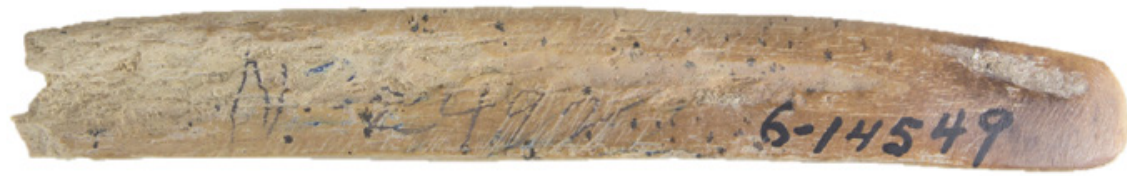

Broken

Exterior Surface

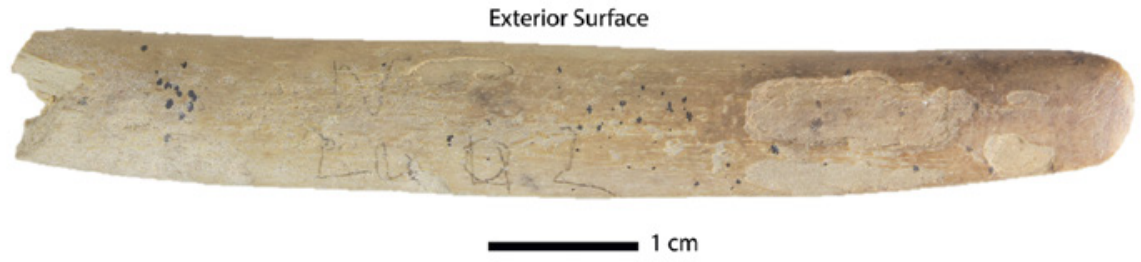

FIGURE 6.1 Bone chisel (6-14549) with tool parts discussed in text labelled (exterior surface, interior surface, beveled end, broken/unmodified end)

$\mathrm{N}_{2492}$ (TC) Map $\mathrm{C}_{4}$

[ed.: According to Hearst Museum records, PAHMA 6-14549 is from this burial.] (Fig. 6.1)

(1) PAHMA 6-14549 Chisel

Material: Bone.

Measurements: $7.5 \mathrm{~cm}$ length, $1 \mathrm{~cm}$ width, $0.5 \mathrm{~cm}$ thickness (max).

This piece is a bone chisel (not ivory as also listed in the museum collections). It is a split long bone fragment (medium mammal, unknown taxa or element), with one end beveled flat (Figure 6.1). It is highly polished on both surfaces and along its margins, but the polish is best developed predominantly at the tip of the chisel (with the other end broken and showing very light polish; this break is fresh and post-excavation). The outer/exterior surface of the bone has flaked off in some areas due to post-excavated drying and treatment, especially on the outer surface, which also exhibits small black marks across its surface (mold?). One end is beveled flat (the "chisel" portion). Both sides, but especially the exposed interior of the bone fragment, show traces of manufacturing under the polish in the form of striations from abrasion. The striations run oblique/diagonal on the interior surface of the bone; polish and beveling is much more extensive here too. The marrow cavity of the bone is visible on this surface, and there is evidence of gouging to remove bone here to shape this surface. The exterior surface of the bone shows only some oblique striations at the tip of the piece and, in fact, it is unclear whether these striations here result 
from manufacturing or use. The margins are highly polished, with one edge rounded and the other comparatively pointed. The rounded edge also shows more extensive polishing and edge fracturing towards the pointed end than the pointed edge shows, suggesting that the rounded edge was more extensively used. The tip remains quite sharp. There is a small piece of bone removed from the interior side of the tip and another along one margin of the chisel near the tip that may represent gouges from use. The tip of the bone is a darker brown to reddish on both surfaces and margin, suggestive of heat treatment and extensive use (given the extent of polish development here). There are recent pencil marks on both surfaces of the tool and an accession number written in marker on the interior surface.
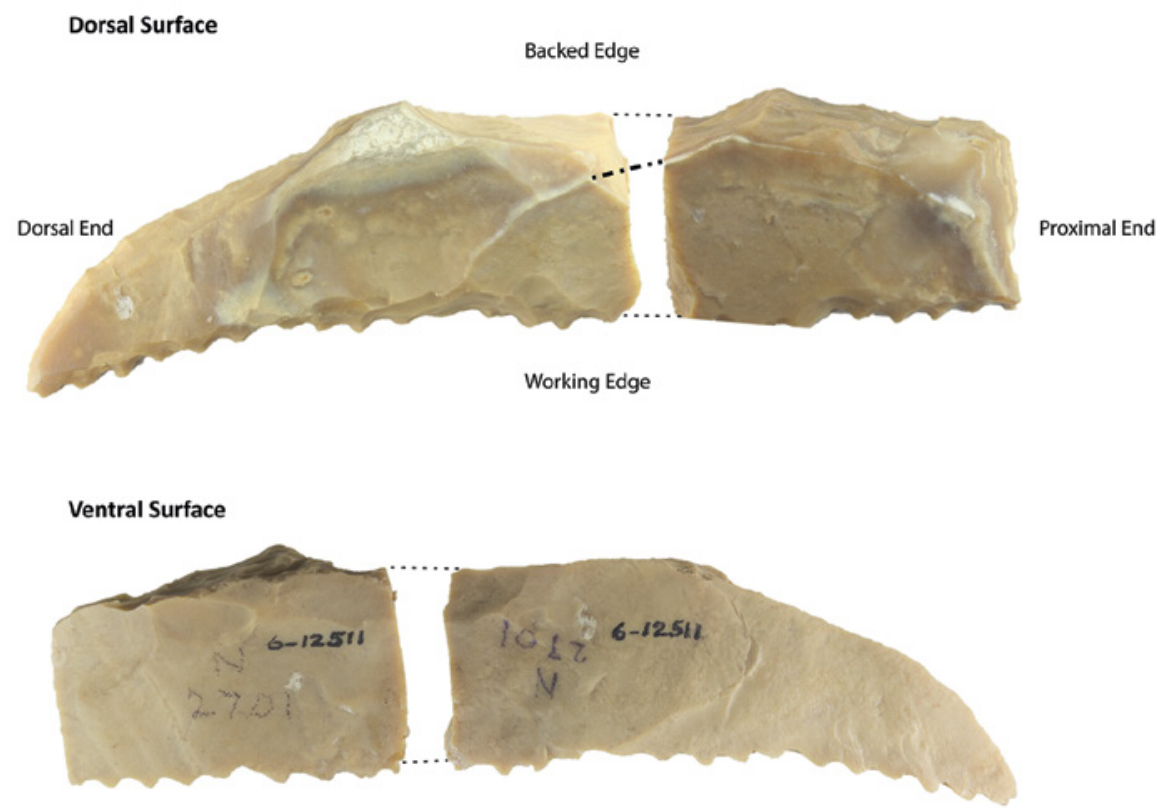

FIGURE 6.2 Reconstructed joining of two knife fragments (6-12511) with tool parts discussed in text labelled (backed edge, working edge, tip or distal end, base or proximal end)

N 2701 (Green) (TC) Map C4

[ed.: According to Hearst Museum records, PAHMA 6-12511, PAHMA 6-12513 are from this burial.] (Fig. 6.2)

(1) PAHMA 6-12511 Broken knife

Material: Flint.

Measurements: $12 \mathrm{~cm}$ length, $3 \mathrm{~cm}$ width, $1.5 \mathrm{~cm}$ thickness (max). 
This object is actually two conjoining pieces of banded flint that were broken after excavation and collection. The break is fresh, and there is glue residue in the break indicative of an attempt to refit and conjoin the two pieces in the past. There is also a small chip/fragment of the edge of one piece missing from the break, further suggesting that the attempt to reattach the two pieces results from an accidental break occurring after excavation. This piece was likely collected intact, and the break did not happen in antiquity - thus, disposal of the piece in antiquity was not a result of the structural failure of the tool (Figure 6.2). Since there is no doubt about these two fragments belonging to the same original single knife, further reference to "the piece" refers to both fragments together.

This piece is manufactured on a medium-grained, slightly brown-banded, tan-colored flint, with traces of a white chalk or marly limestone cortex on both the dorsal and ventral surfaces of both pieces. The largest exposure of visible cortex (dorsal surface of larger, pointed fragment) shows a visibly pitted surface characteristic of extensive wind abrasion. Rare concentric, crystalline inclusions are visible on the dorsal surface of the pieces; the inclusions may have hindered the workability of the flint, particularly in attempts to thin the blade (see below).

The "knife" is manufactured from a blade blank; however, perpendicular flake scars on the dorsal surface suggest that this blade blank could have been removed from either a primarily flake-based or blade-based core reduction technology, with recent flake removals during core maintenance. The proximal and distal ends of the piece have been extensively modified by retouch. However, the natural tapering and thinning of the pointed end of the tool (seen also in the direction of blade scars) and thicker butt end of the tool, provide clear indications of the pieces' orientation, with the dorsal end terminating in a point and the blunted butt end of the piece being the proximal end. It would have measured approximately $9-10 \mathrm{~cm}$ in total length (reconstructed from conjoining the two pieces) and averaged no more than $5 \mathrm{~cm}$ in width. The tool varies considerably from the proximal to distal ends, with the proximal end measuring $9 \mathrm{~mm}$ in thickness, the middle of the piece measuring $12 \mathrm{~mm}$ in thickness, and the distal (pointed) end of the piece measuring $4 \mathrm{~mm}$ in thickness.

The piece evidences traces of several failed attempts to thin the blade, resulting in many hinged and stepped fractures along the backed edge. After extensively hinging the dorsal surface of the piece, the knapper abandoned further attempts to thin the piece and modified the tool's edges as is. The piece, thus, has a steep, triangular cross-section, especially towards its proximal end. This failure of the knapper to adequately thin the blade (evidenced by hinged 
terminations in the absence of raw material inclusions or other flaws) suggests some degree of inexpertness in knapping skill.

The backed edge of the piece shows an abrupt, curved profile as a result of shaping by steep, large, abrupt retouch scars that run discontinuously along this edge. There is one small area along the backed edge of the larger, pointed distal fragment of the knife that is left unretouched. The entire edge of the smaller fragment is extensively backed, with the backing overprinting larger hinged flake scars from failed attempts to thin the piece (especially at its midpoint). The larger, pointed fragment shows similar overprinting at its midpoint, while the distal end is tapered to a point as a result of the backing. The distal end of the piece is thus pointed; the proximal end is retouched with a straight truncation.

The working edge of the piece has been heavily denticulated, where the knapper manufactured several large notches or serrations along the entire edge of the piece. This serrated working edge also exhibits a slightly concave profile from proximal to distal ends, notable since the cross-section profile of the piece is largely straight (although the piece bulges slightly in thickness at midpoint). Notably, the working edge shows clear evidence of polish. Although use-wear analysis was not performed on this piece, the polish is similar to sickle polish documented elsewhere (Macdonald 2014; Richter 2007; UngerHamilton 1988) that results from contact with silica-rich grasses. This polish is only documented on the highest ridges of the serrations along the worked edge and was not noted in the "grooves" of the notch flake scars (Figure 6.3).

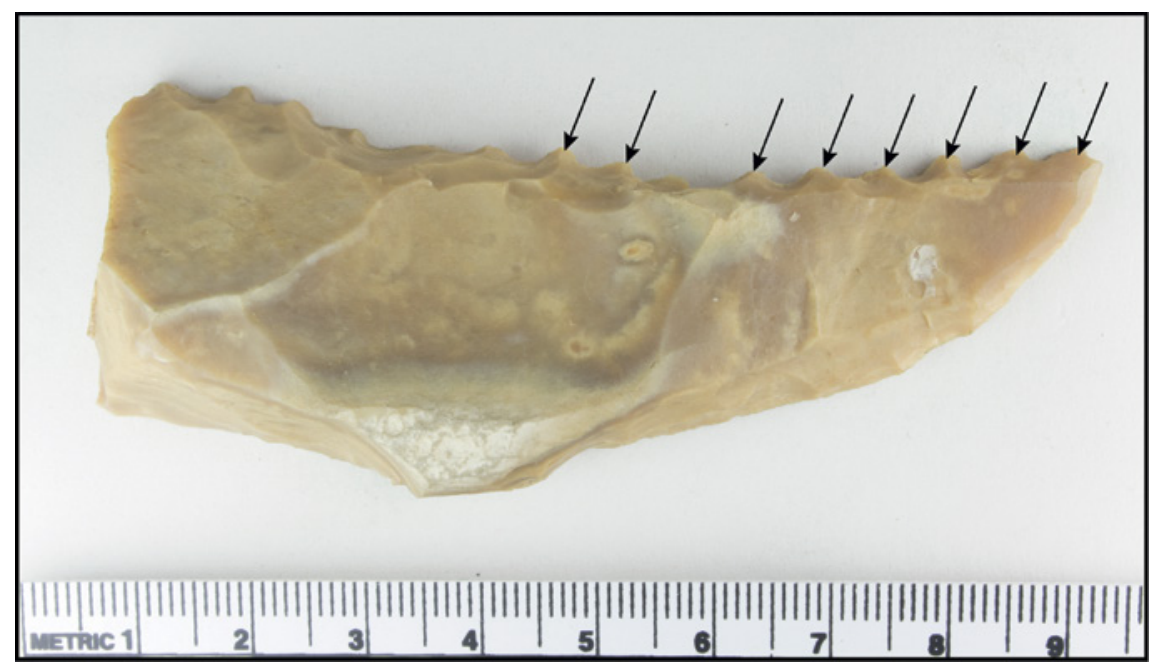

FIGURE 6.3 Close-up of the dorsal surface of the working edge of 6-12511, with arrows showing the locations of visible polish 
All of the retouch (the backing along the backed edge and the notches produced to denticulate the working edge) is unilateral and visible only on the dorsal surface of the knife. The ventral surface is highly fractured, showing a coarseness to the raw material.
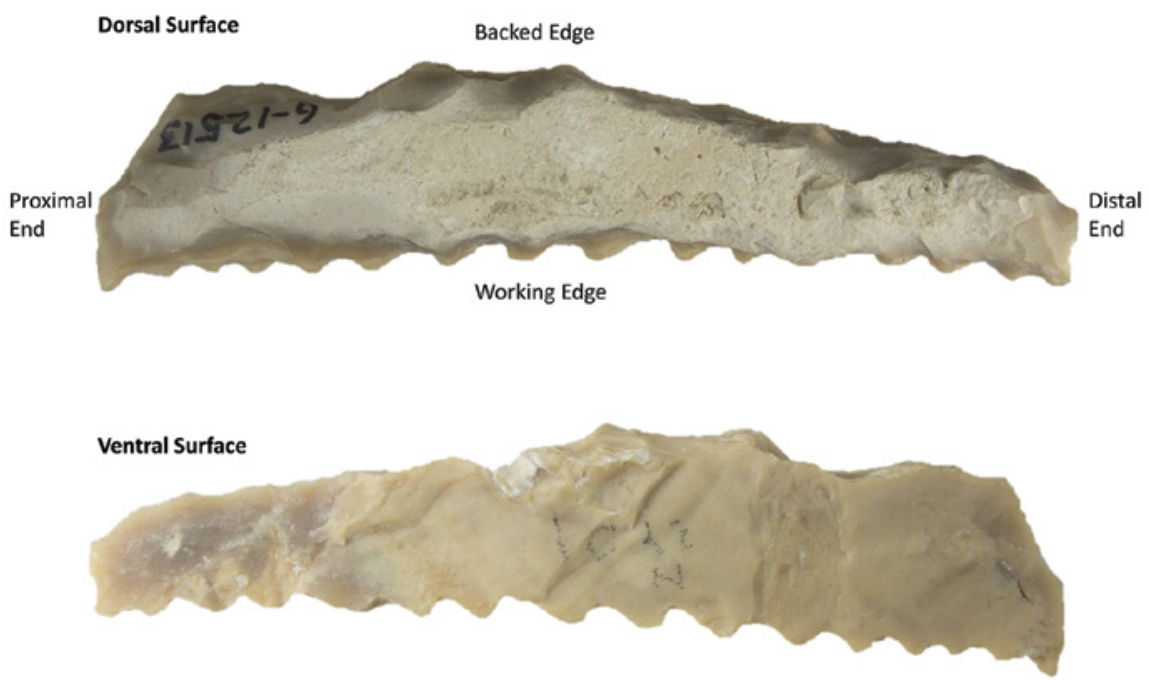

FIGURE 6.4 Schematic of the almost complete knife (6-12513) with tool parts discussed in text labelled (backed edge, working edge, tip or distal end, base or proximal end, ventral surface, dorsal surface)

(2) PAHMA 6-12513 Knife

Material: Flint.

Measurements: $8.5 \mathrm{~cm}$ length, $1.4 \mathrm{~cm}$ width, $0.8 \mathrm{~cm}$ thickness (max).

This object is reported as a complete knife manufactured from a similar raw material to PAHMA 6-12511 (the above-mentioned broken knife) (Figure 6.4). It is a banded light brown flint of a similar medium-to-coarse quality as PAHMA 6-12511. The bands are a darker brown, and there are rare concentric crystalline inclusions throughout the piece. The piece also exhibits some traces of the rock's original cortex (weathered outer surface); it is a white chalk or marly limestone. The abundance of crystalline inclusions and very rough, uneven, and fractured ventral surface suggests a relatively poor quality flint, and this would have impacted its ability to be easily knapped. Indeed, a crosssection view of the piece indicates that the flint easily fractures in a laminar manner that follows some of the visible banding and inclusions, and this may account for the extensiveness of stepped fractures produced during edge 
retouching. Thus, the success in making the knife suggests its manufacturer was not a novice (although the abundance of hinged and stepped terminations along the backed edge suggest the knapper experienced challenges in thinning this piece too). Although petrographic or geochemical examination of the raw material was not performed, macroscopic visual comparison strongly suggests that these two pieces (PAHMA 6-12511 and PAHMA 6-12513) were manufactured on flint from the same geological outcrop (even though this knife was made on a coarser variant of this flint).

One end of the small elongated piece has been retouched to create an end that curves towards the backed edge, while the other end, which tapers considerably, is broken. The tapering evident towards this end suggests that it may have been pointed, as with PAHMA 6-12511, and just the tip is missing. This break is not as evidently recent as PAHMA 6-12511 (and there are no traces of attempted repair), and so the piece may have been excavated as is, with the tip broken in antiquity.

The "knife" is manufactured from a blade blank. The extensiveness of cortex on the dorsal surface of the piece indicates that it was produced on either a primary piece with extensive edge modification or, more likely, a secondary blade with cortex remaining in the center of the dorsal surface. Given the coarseness of the raw material and its propensity to laminar fracturing, the knapper did not invest much effort in thinning the piece, resulting in an overall thick, triangular profile and a dorsal surface covered in cortex.

It is less clear which "end" of the knife represents the proximal or distal ends of the blade blank, as there are no clear ripple marks on the ventral surface or traces of a bulb of percussion, platform, point of impact, or eraillure scars. One end is extensively retouched to produce a curved profile, curved towards the backed edge. The other end is broken. As the retouched end is wider and thicker and the broken end tapers towards a (broken) point, it is likely that the retouched end is the proximal end and the broken tip is the distal end of the blade. The knife is $80 \mathrm{~mm}$ in maximum length.

The backed edge of the piece shows an abrupt, curved profile as a result of shaping by steep, large, abrupt retouch scars that run continuously along this edge. This edge is notably thicker than the working edge and covered in scars with stepped and hinged terminations that represent a combination of attempts to thin the blade and retouch scars from backing.

Like PAHMA 6-12511, the working edge of the piece has been heavily denticulated, where the knapper manufactured several large notches or serrations along the entire edge of the piece. This serrated working edge has a straight profile from proximal to distal ends. The working edge shows no clear evidence of polish or any other forms of use. All of the retouch (the backing along the 
backed edge and the notches produced to denticulate the working edge) is unilateral and visible only on the dorsal surface of the knife. The ventral surface is highly fractured, showing a coarseness to the raw material.

\section{Bibliography}

Macdonald, D. A. 2014. "The Application of Focus Variation Microscopy for Lithic Use-Wear Quantification." Journal of Archaeological Science 48:26-33.

Richter, T. 2007. "A Comparative Use-Wear Analysis of Late Epipalaeolithic (Natufian) Chipped Stone Artefacts from the Southern Levant." Levant 39:97-122.

Unger-Hamilton, R. 1988. Method in Microwear Analysis: Prehistoric Sickles and Other Stone Tools from Arjoune, Syria. BAR International Series 435. Oxford. 